\title{
La nécropole romaine de Porta Nocera à Pompéi. Campagne 2017
}

William Van Andringa, Thomas Creissen et Henri Duday

\section{(2) OpenEdition \\ Journals}

Édition électronique

URL : http://journals.openedition.org/cefr/1946

DOI : 10.4000/cefr. 1946

ISSN : 2282-5703

Éditeur

École française de Rome

Référence électronique

William Van Andringa, Thomas Creissen et Henri Duday, «La nécropole romaine de Porta Nocera à Pompéi. Campagne 2017 », Chronique des activités archéologiques de l'École française de Rome [En ligne], Les cités vésuviennes, mis en ligne le 19 juillet 2018, consulté le 20 novembre 2019. URL: http://journals.openedition.org/cefr/1946; DOI : 10.4000/cefr.1946

Ce document a été généré automatiquement le 20 novembre 2019.

(c) École française de Rome 


\title{
La nécropole romaine de Porta Nocera à Pompéi. Campagne 2017
}

\author{
William Van Andringa, Thomas Creissen et Henri Duday
}

\section{NOTE DE L'ÉDITEUR}

La fouille de la nécropole de Porta Nocera fait partie du programme de recherches « Porta Nocera " porté par l'École française de Rome, l'université de Lille 3 (Centre de recherches Halma - UMR 8163 du CNRS), la société archéologique Éveha International en collaboration avec l'Institut Universitaire de France, l'Institut archéologique autrichien de Vienne (ÖAI) et le soutien du Parco archeologico di Pompei. À ce titre, nous tenons à remercier le professeur Massimo Osanna, directeur du Parco, et le dott. Fabio Galeandro ainsi que Catherine Virlouvet, directrice de l'École française de Rome et Nicolas Laubry, directeur des Études, pour leur soutien. Cette chronique résume les résultats exposés dans le rapport d'opération rédigé par l'ensemble des membres de l'équipe cités dans la note ci-dessous et déposé au Parco archeologico di Pompei et à l'École française de Rome : W. Van Andringa, Th. Creissen, H. Duday (dir.). Naissance et développement d'un paysage funéraire romain (IIr siècle av. $-I^{e r}$ siècle apr.J.-C.). Aux portes de la ville et le long de la route de Nocera. Fouille de deux secteurs de la nécropole romaine de Porta Nocera à Pompéi, Rapport d'opération, Pompéi, 2017, 620 p.

\section{NOTE DE L'AUTEUR}

La campagne 2017 a réuni une équipe d'une quarantaine de chercheurs, professionnels et doctorants : Marie-José Ancel (Archeodunum), Reine-Marie Bérard (EFR), Antoine Boisson, Alexandra Boucherie (ULB), Philippe Brunner, Cécilia Cammas, Marie-Caroline Charbonnier (Inrap), Anselme Cormier, Thomas Creissen (Evéha International), Sandra Dal Col, Franck Decanter (Inrap), Elsa Dias (Archeodunum), Jean-Patrick Duchemin (université de Lille 3), Henri Duday (université de Bordeaux), Aude Durand (université de 
Lille 3), Arnaud Février (ESTP), Klaus Freitag (Istituto archeologico di Vienna), Flore Giraud, Stefan Groh (Istituto archeologico di Vienna), Alexis Jaubron (ESTP), Johannes Laiho, Aurore Lambert (Evéha), Alexia Lattard, Tuija Lind, Claude Malagoli, Adrien Malignas, Véronique Matterne (CNRS), Pascal Neaud (Inrap), Clarisse Parra-Prieto, Émilie Portat (Direction de l'Archéologie, Mairie de Chartres), Ivan Repetto (Istituto archeologico di Vienna), Laudine Robin (Evéha), Géraldine Sachau-Carcel, Sophie Thorimbert (Archeodunum), William Van Andringa (université de Lille 3 et IUF), AnneSophie Vigot (Evéha).

1 Amorcé en 2014, le nouveau programme d'étude de la nécropole de Porta Nocera a pour première ambition de comprendre et de caractériser les processus de constitution d'un paysage funéraire aux abords de la ville entre la fondation de la colonie et l'éruption de 79 , notamment l'implication des autorités publiques dans la planification et la gestion des aires funéraires. Il s'agit ensuite, sur un temps relativement court -quelques générations, de préciser la genèse des différents enclos et structures funéraires (chronologie relative des tombes) et de reconnaître des traditions familiales ou de groupes dans l'aménagement des sépultures et dans l'exercice des séquences rituelles. Enfin, l'exceptionnel état de conservation des ensembles funéraires permet d'étudier au plus près les trois grandes étapes des rituels de la mort que sont la crémation, la mise au tombeau et la commémoration des défunts. À chacune de ces étapes, il est désormais possible d'associer des gestes très précis dont la compilation permettra de proposer une forme d'herméneutique fondée sur la lecture attentive du terrain. Dans ce domaine, le programme comporte une dimension méthodologique forte, autant sur la fouille, dans la mise en place des protocoles d'enregistrement spécifiques destinés à reconnaître les gestes, qu'en laboratoire avec l'étude des restes humains brûlés et du mobilier utilisé dans les séquences rituelles. La richesse des faits observés permet d'ores et déjà de proposer des résultats inédits et déterminants sur la structure des rites déployés dans les enclos ainsi que sur l'élaboration et la transmission des coutumes funéraires au sein d'une communauté romaine d'Italie (Duday - Van Andringa 2017). Comment étaient organisées et transmises les partitions rituelles d'une génération à l'autre, d'une famille à l'autre? Comment était construite l'altérité collective de la mort à Pompéi ? En quoi les pratiques suivaient-elles la tradition romaine? Un enregistrement aussi exhaustif que possible de toutes les traces laissées par les Pompéiens lors de leur fréquentation de la nécropole amène désormais à distinguer l'occasionnel de l'intentionnel pour restituer le contenu des pratiques funéraires locales. L'investissement méthodologique et les résultats obtenus justifient pleinement l'organisation d'une formation en archéologie funéraire destinée à des doctorants et professionnels, qui concerne tout autant la fouille des structures funéraires que l'étude spécifique des crémations. Lors de la campagne 2017, un stage dirigé par l'un de nous (H.D.) a réuni 8 personnes qui ont pu se former à l'étude des os brûlés à partir des tombes exhumées au cours des précédentes campagnes de fouilles, avec une recherche plus particulièrement orientée vers les liaisons ostéologiques entre différentes unités stratigraphiques et/ou fonctionnelles (fig. 1). 
Fig. 1 - Analyse des os brûlés.

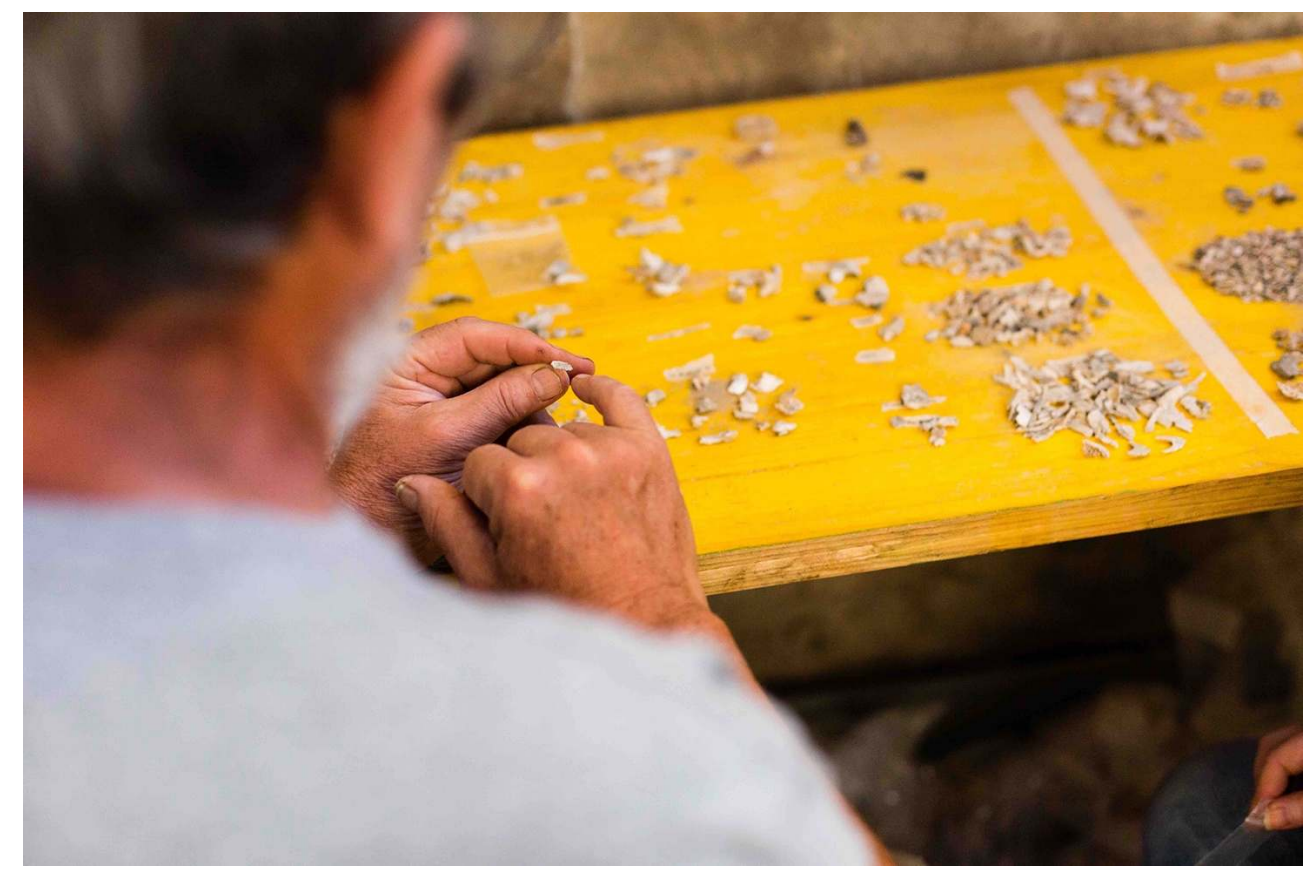

Cl. F. Giraud/@ MAPN. Toute reproduction, par quelque moyen que ce soit, reste interdite.

2 La campagne 2017 s'est déroulée entre le 21 août et le 30 septembre, sur deux zones de la nécropole : en partie ouest, la zone A, située dans le prolongement du quartier étudié entre 2003 et 2007 (Van Andringa et al. 2013) et en partie est, la zone B, une section de la nécropole dégagée en 1983 (D’Ambrosio - De Caro 1987) (fig. 2). En zone A, l'étude du monument 26a et de la voie se dirigeant vers la porte de Stabies s'est poursuivie. Il s'agit d'un enclos à ciel ouvert, sans accès direct, dont le mur de fond était sommé d'un fronton abritant une niche centrale portant l'urne de verre de la titulaire de l'enclos, Poppaea Cypare. L'intérieur est désormais intégralement fouillé pour ce qui concerne les niveaux contemporains de sa construction ou postérieurs à celle-ci. Un bûcher antérieur à l'aménagement de l'enclos - sans lien avec celui-ci - a été partiellement observé dans un sondage profond (fig. 3). Dans l'enclos, trois nouvelles tombes ont été fouillées en 2017. Parmi celles-ci figure une tombe qu'une stèle en marbre attribue à une enfant, Aphe, morte à 6 ans (SP 309). À cette stèle était associée une urne en céramique contenant quelques os brûlés ainsi qu'un petit vase en verre lui aussi rempli d'os brûlés. L'étude ostéologique a pu montrer que les deux récipients contenaient les restes de deux individus immatures, l'un âgé d'environ 3 ans, l'autre d'environ 6 ans. La stèle qui signale une enfant morte à 6 ans se rapporte donc au second individu, mais les fragments osseux appartiennent majoritairement au sujet le plus jeune. À proximité de cette tombe, la sépulture SP 313 ne possédait probablement pas de marquage de surface. Le contenant funéraire est une urne en céramique dans laquelle avait été déposé un sac contenant les os brûlés, une clochette en bronze et, probablement, de l'encens brûlé. Une monnaie surmontait le sommet de l'amas. La dernière tombe (SP 312) se trouve en pendant. Son emplacement était marqué par un bloc de lave à peine équarri et le négatif d'un conduit à libations en matériau périssable a été identifié (à moins que le négatif corresponde à un tube à libations prélevé). Les ossements brûlés étaient rassemblés dans une urne en céramique. En attendant l'étude anthropologique, l'observation des ossements permet 
d'identifier un adulte. La fouille de SP 307, amorcée en 2016, s'est poursuivie. À la stèle est associé un dispositif à libations fonctionnant avec l'enclos 26a dont il a été possible de démontrer qu'il est venu se superposer à un aménagement plus ancien, en partie coupé par les murs de l'enclos. Il avait été envisagé qu'il s'agissait d'une tombe appartenant à un premier état de ce lot funéraire qui aurait été réaménagée lors de la construction de l'enclos actuel. La campagne de 2017 a démontré qu'il s'agissait bien d'une structure funéraire, en l'occurrence un premier dispositif à libations : aucun dépôt osseux associé à la SP 307 n'est présent à l'intérieur de l'enclos.

Fig. 2 - Vue aérienne de la nécropole de Porta Nocera.

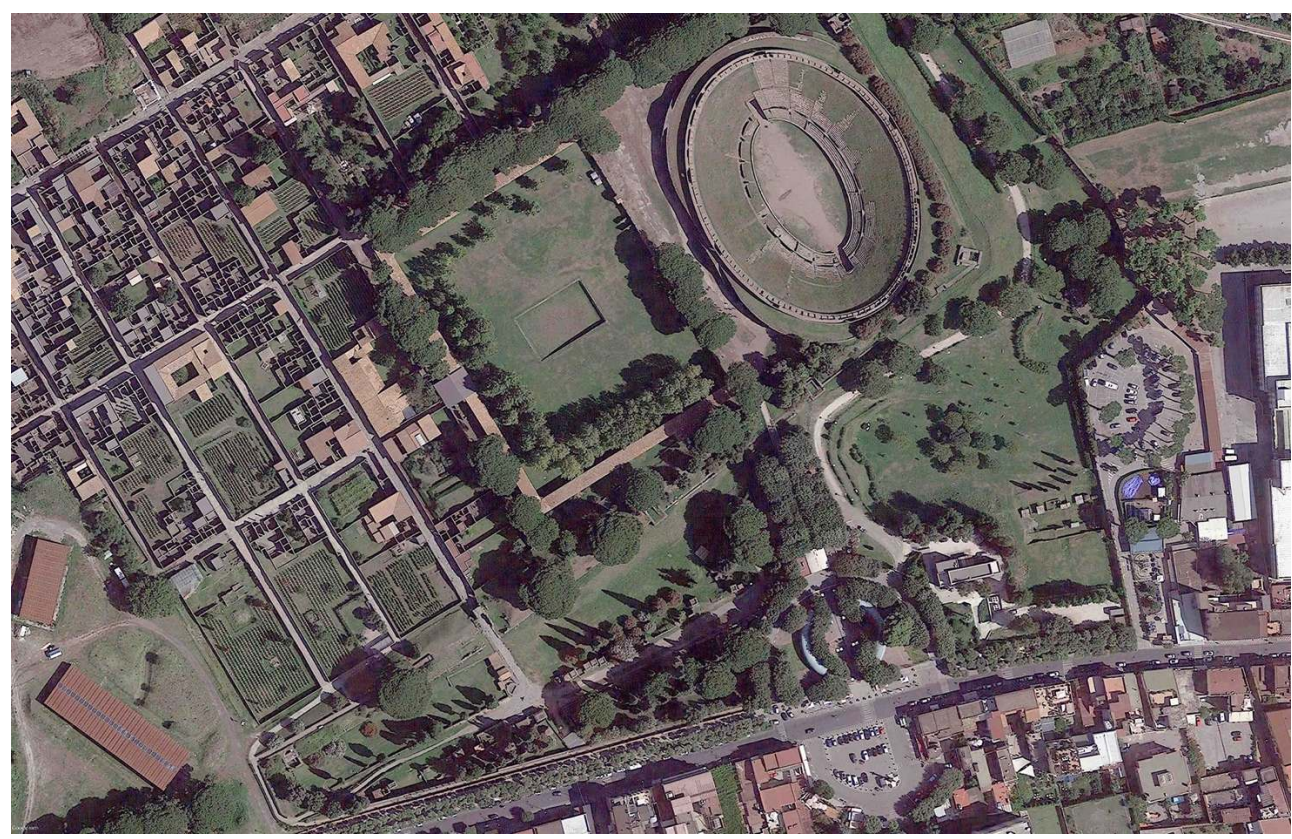

(c) MAPN. Toute reproduction, par quelque moyen que ce soit, reste interdite. 
Fig. 3 - Bûcher antérieur à l'aménagement de l'enclos 26a.

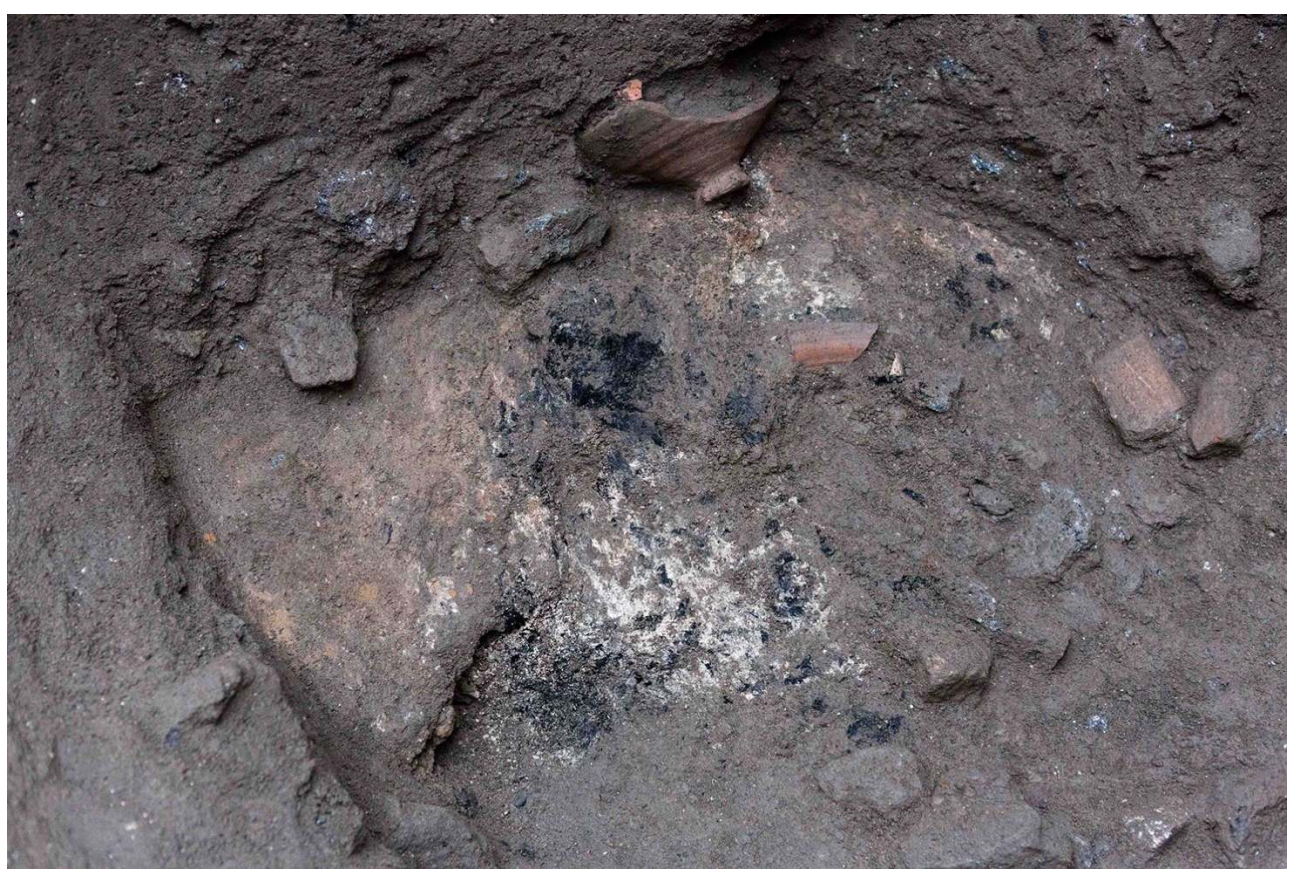

CI. F. Giraud/@ MAPN. Toute reproduction, par quelque moyen que ce soit, reste interdite.

3 L'étude ostéologique des restes associés à la tombe 311 a livré des informations déterminantes sur la genèse de l'enclos. Dans un premier temps, cette tombe a abrité les os brûlés de l'individu dont une partie des restes a ultérieurement été " mise en scène » dans l'urne en verre qui a alors été placée dans la niche du fronton. Selon toute vraisemblance, Poppaea Cypare - le nom inscrit sur la stèle en marbre associée à la tombe au sol - est décédée avant la construction de l'enclos et ses os brûlés ont été déposés dans une première tombe (SP 311), à l'intérieur d'un contenant non identifié - un sac ou une urne ?. Puis ses os ont été exhumés et placés dans la niche une fois le monument achevé. La stèle associée à la tombe 307 a elle aussi été installée au moment de l'achèvement de l'enclos, lors de la mise en place des sols. Elle correspond à une sépulture antérieure, dont une partie du dispositif de libations a été entaillée par la construction de l'enclos. L'absence de dépôt osseux associé amène à formuler deux hypothèses : soit il s'agit d'un cénotaphe; soit la sépulture est située un peu plus au sud, hors l'emprise de l'enclos actuel. Dans le second cas, l'emprise du lot funéraire aurait quelque peu évolué au cours du temps.

$4 \mathrm{Au}$ total, 7 tombes sont présentes à l'intérieur de l'enclos. L'une pourrait être vide ; une autre contient les os de deux individus et une dernière possède deux marquages distincts: un dispositif funéraire classique au sol et une niche aménagée dans la maçonnerie accueillant une urne en verre.

Dans le secteur de la voie bordant l'enclos, la fouille est descendue jusqu'au socle rocheux en lave, entaillé de nombreux creusements du fait de la préexistence d'une carrière. Après son abandon, celle-ci s'est progressivement comblée avant que l'ensemble ne soit remblayé et partiellement aplani de manière à aménager la première chaussée. Au-dessus de celle-ci, quelques recharges se rapportent aux plus anciennes utilisations de la voie, encore mal datées. C'est sur ces niveaux qu'est construit l'enclos 26a, directement adjacent à la voie, dont les angles nord-est et nord-ouest étaient protégés par un bloc de 
lave (chasse-roues). Progressivement, des recharges de voierie se sont accumulées contre les murs de l'enclos. C'est à la surface de celles-ci que se trouvait une succession de dépôts de restes alimentaires brûlés ( 9 occurrences) ou de concentrations de charbons de bois (2 occurrences). La nature des dépôts est très variée (fig. 4). Il s'agit principalement de végétaux (figues, noix, olive, raisin, pain...), mais des restes animaux sont également présents : le porc domine largement mais du mouton, des moyens passereaux, du coq ainsi que des restes de poissons et d'oursins sont également attestés. La récurrence de ces dépôts en un même endroit, dans l'axe central de l'enclos 26a, invite à les associer à ce dernier. Il pourrait s'agir d'offrandes alimentaires qui auraient été brûlées directement sur le talus bordier de la route, à l'extérieur du monument, du fait sans doute de la difficulté d'accès à l'intérieur de l'enclos (absence de porte ou d'escalier ménagé dans la maçonnerie). Un officiant serait ainsi venu célébrer à neuf reprises au moins le souvenir de Poppaea Cypare et des siens, avant que le monument soit progressivement abandonné et envahi par des couches d'alluvions.

Fig. 4 - Route et talus bordant l'enclos 26a. La couche charbonneuse garde le souvenir d'offrandes alimentaires brûlées en hommage aux défunts.

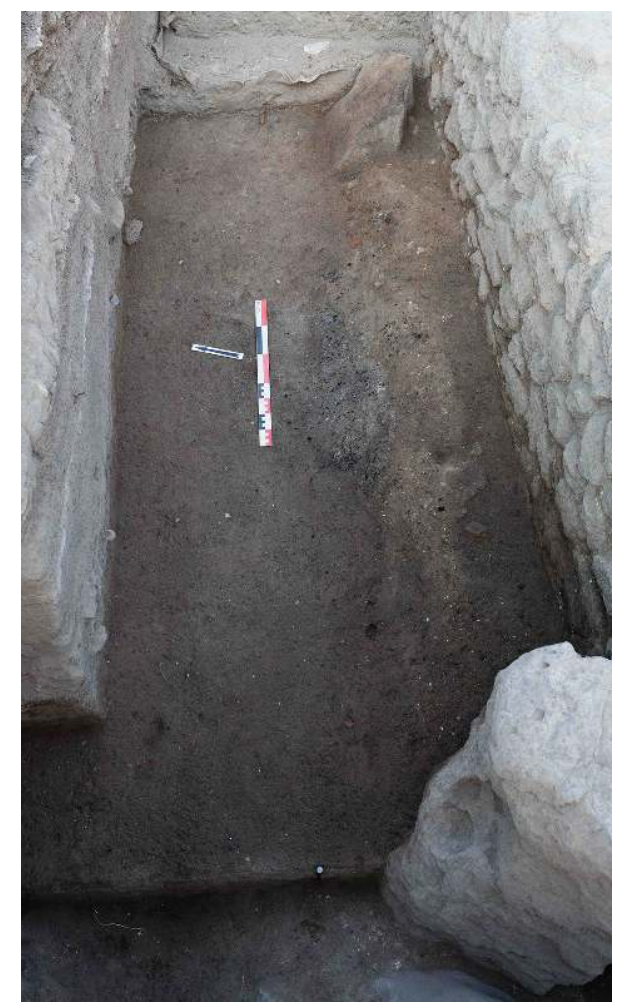

Cl. F. Giraud/@ MAPN. Toute reproduction, par quelque moyen que ce soit, reste interdite.

6 En zone B, les travaux ont porté sur la route de Nocera et sur certains enclos funéraires qui bordent celle-ci au nord et au sud (fig. 5). Pour la voie, de nouvelles zones de fouilles ont été ouvertes dans l'espoir de trouver du mobilier permettant d'en préciser la datation (fig. 6). L'analyse des trottoirs et des accotements s'est poursuivie dans un double objectif : comprendre leurs liens avec les monuments funéraires voisins, et déterminer si leur entretien et leur aménagement était plutôt le fait des propriétaires des enclos ou plutôt celui des autorités de la cité. Autrement dit, s'agissait-il d'espaces publics ou privés ? La grande nouveauté de cette campagne réside dans la mise en évidence, au sein 
de deux sondages profonds, d'un premier état de chaussée qui se développait sous les monuments funéraires, c'est-à-dire plus au nord. La voie actuelle a été aménagée dans un second temps, moyennant un nivellement de la surface du rocher et d'importants travaux de terrassement. Le remblai issu de ce chantier aurait été utilisé pour constituer les talus bordiers ainsi que les terrasses qui bordent la voie, aujourd'hui encaissée. C'est au sommet de ces remblais que les lots funéraires encore visibles ont été aménagés, ce qui pourrait indiquer que la première voie est antérieure à la constitution de la nécropole.

Fig. 5 - Photogrammétrie de la zone B en cours de fouille.

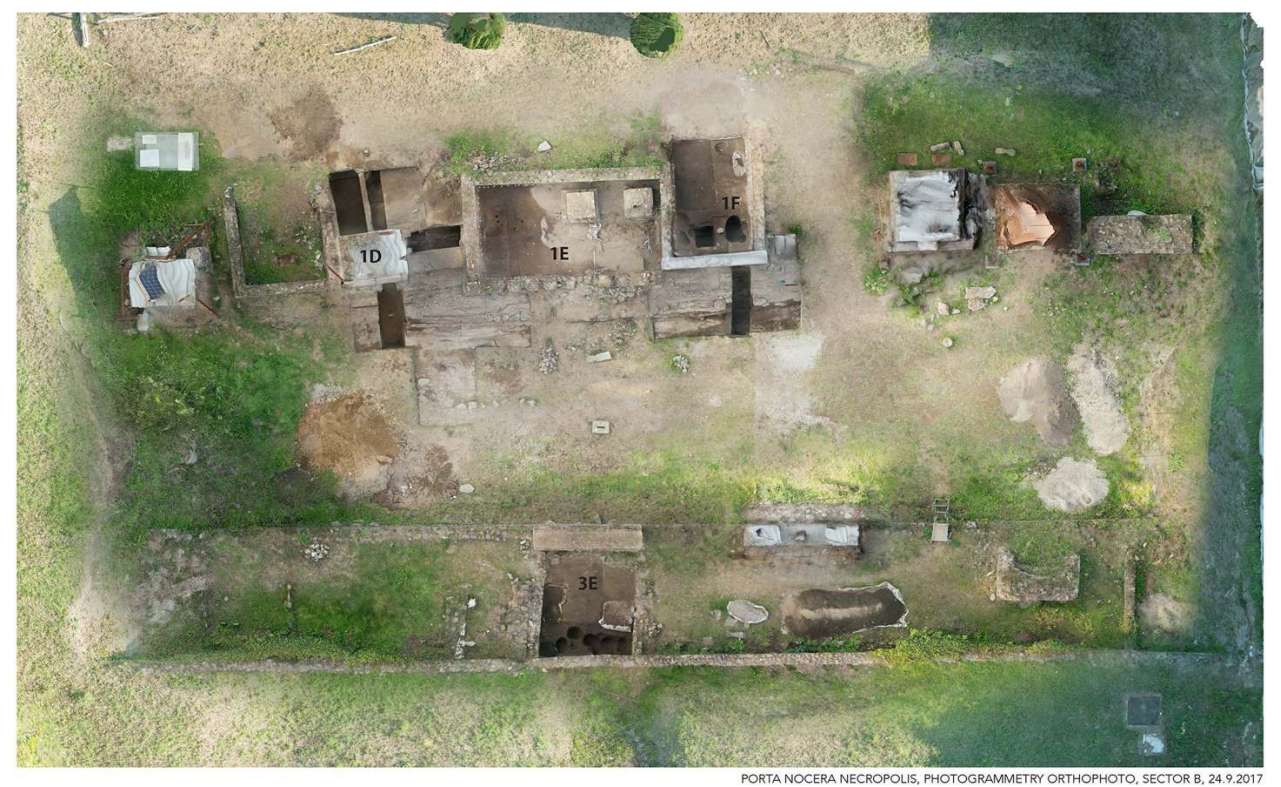

Cl. J. Laiho/@ MAPN. Toute reproduction, par quelque moyen que ce soit, reste interdite. 
Fig. 6 - Talus et trottoir bordier devant le monument 1D. On note l'aménagement d'un sol de galets uniforme devant l'ensemble des monuments et des enclos, témoignage d'une mise en place programmée et publique des aires funéraires.

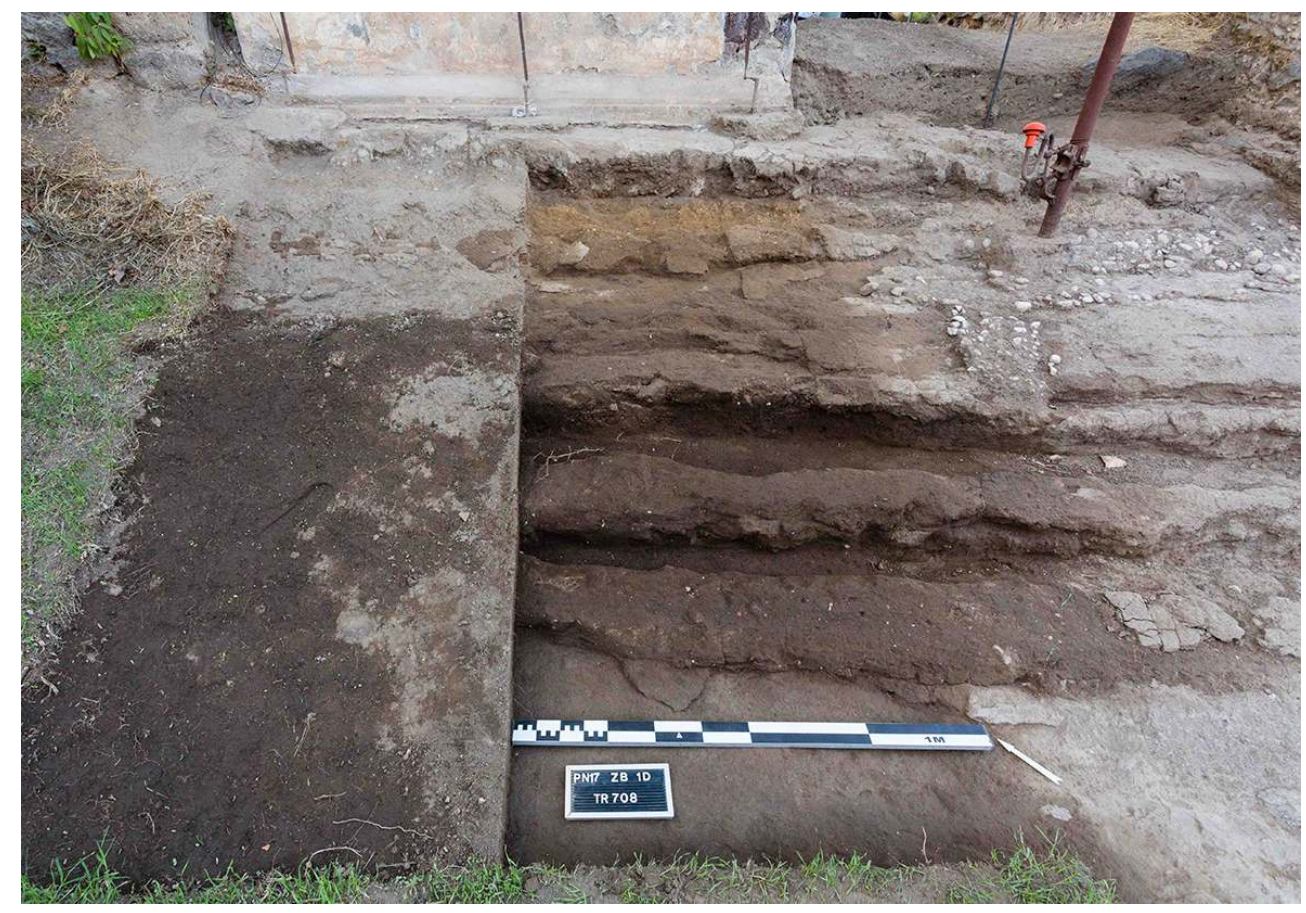

Cl. F. Giraud/@ MAPN. Toute reproduction, par quelque moyen que ce soit, reste interdite.

7 Au nord, un nouvel enclos a dû être étudié cette année dans le cadre des projets de conservation de la nécropole ${ }^{1}$. Il s'agit de l'enclos 1D dont l'épigraphie nous indique qu'il était destiné à un couple d'affranchis : Caecilia L(uci) l(iberta) Agathia / viva sibei fecit et / $L$ (ucio) Caecilio L(uci) l(iberto) Dioscuridi / viro suo. Le monument empiète en partie sur l'accotement. Au revers, un étroit couloir est aménagé dans sa partie basse (fig. 7); il donne accès à un petit puits construit dans le soubassement, à l'intérieur duquel une urne en céramique a été déposée. Celle-ci contenait les restes osseux d'un individu âgé de sexe féminin, très certainement Caecilia Agathia. Au-dessus de l'urne, à l'intérieur de la tombe, deux dépôts de grands fragments de gobelets en céramique à parois fines (type Mayet $10 \mathrm{~b} / \mathrm{c}$ ) ont été empilés, vraisemblablement dans le but de commémorer la cérémonie intervenue lors de la mise au tombeau et de la fermeture de la sépulture. La céramique permet de dater la cérémonie en question de l'époque augustéenne ou un peu avant. Une fois les deux piles de vases déposées, un remblai pénétrant dans le couloir est venu sceller l'ensemble pour former un talus au sommet duquel une stèle en lave était jetée (fig. 6, 7011). Cette stèle dépourvue de chignon peut être attribuée à un individu masculin; elle marquait peut-être la sépulture programmée pour Caecilius Dioscurides. L'absence d'une seconde sépulture dans l'emprise du monument montre toutefois que Dioscurides ne fut jamais enterré avec Caecilia Agathia. Sa mémoire fut toutefois maintenue par la dédicace du monument. 
Fig. 7 - Coupe du dépôt funéraire du monument 1D et des remblais de fermeture du tombeau.

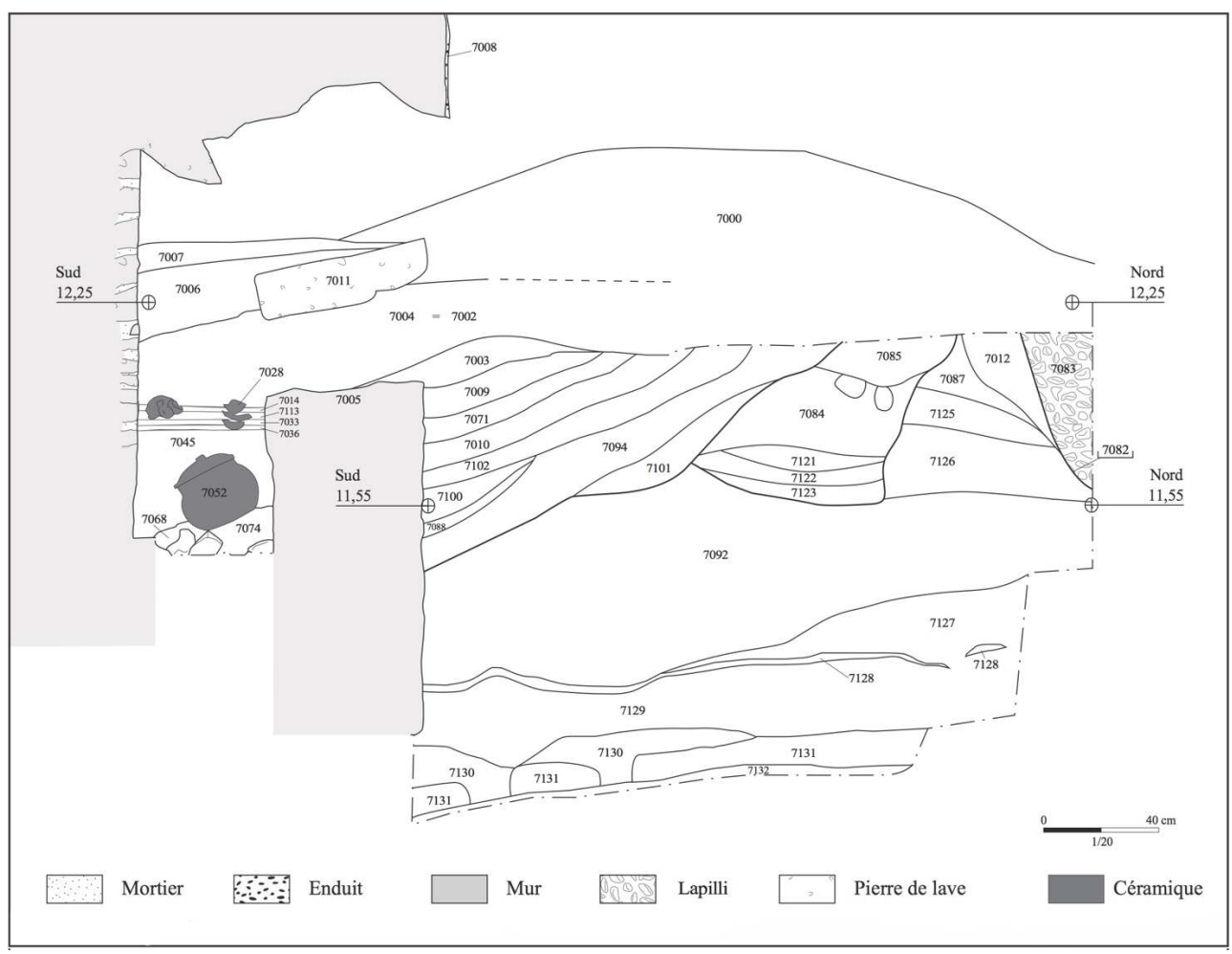

Relevé : M.-J. Ancel, C. Parra Prieto ; DAO : C. Parra Prieto ; @ MAPN. Toute reproduction, par quelque moyen que ce soit, reste interdite.

8 À proximité, l'étude de l'enclos $1 \mathrm{E}$ s'est poursuivie. Celui-ci appartient à une affranchie : Novia C(aii) l(iberta) Amoena / sibi et suis / et L(ucio) Lacellio Virillioni. Des membres extérieurs à la famille autres que Lucius Lacellius Virillio ont été accueillis dans l'enclos comme l'indiquent les stèles élevées sur les tombes de Cornelia Quieta et Cnaeus Turranius Primus. La fouille des deux aires de crémation (AC1 et AC2) installées dans la partie occidentale de l'enclos est maintenant achevée (fig. 8) ; une troisième pourrait exister devant les petits monuments funéraires de Novia Amoena (F310). La fouille des sépultures de l'enclos s'est poursuivie (fig. 9). La tombe SP 1E6 correspond à une sépulture d'immature dont les restes osseux brûlés ont été déposés dans une urne en céramique. Dans l'angle sud-est, la sépulture SP 1E5, appartenant à Cnaeus Turranius Primus, consiste en une fosse dans laquelle avaient été déposés les os brûlés, préalablement rassemblés dans un contenant en matériau périssable. L'ensemble était surmonté d'une mensa associée à une stèle en marbre ainsi qu'à un conduit à libations en céramique. Sous cette tombe, une fosse contenant les os incinérés d'un immature a été identifiée (SP 1E8). Une monnaie y était déposée, qui marquait la constitution de la tombe. Aucun marquage de surface ne peut lui être clairement associé. Peut-être fonctionnait-elle avec la sépulture 5 ? Une inhumation en amphore, partiellement recoupée par des tombes postérieures, n'a pas pu être intégralement fouillée (SP 1E7) parce qu'elle est engagée sous la maçonnerie du monument de Novia Amoenia. Elle correspond à un individu périnatal. 
Fig. 8 - L'aire de crémation AC 2.

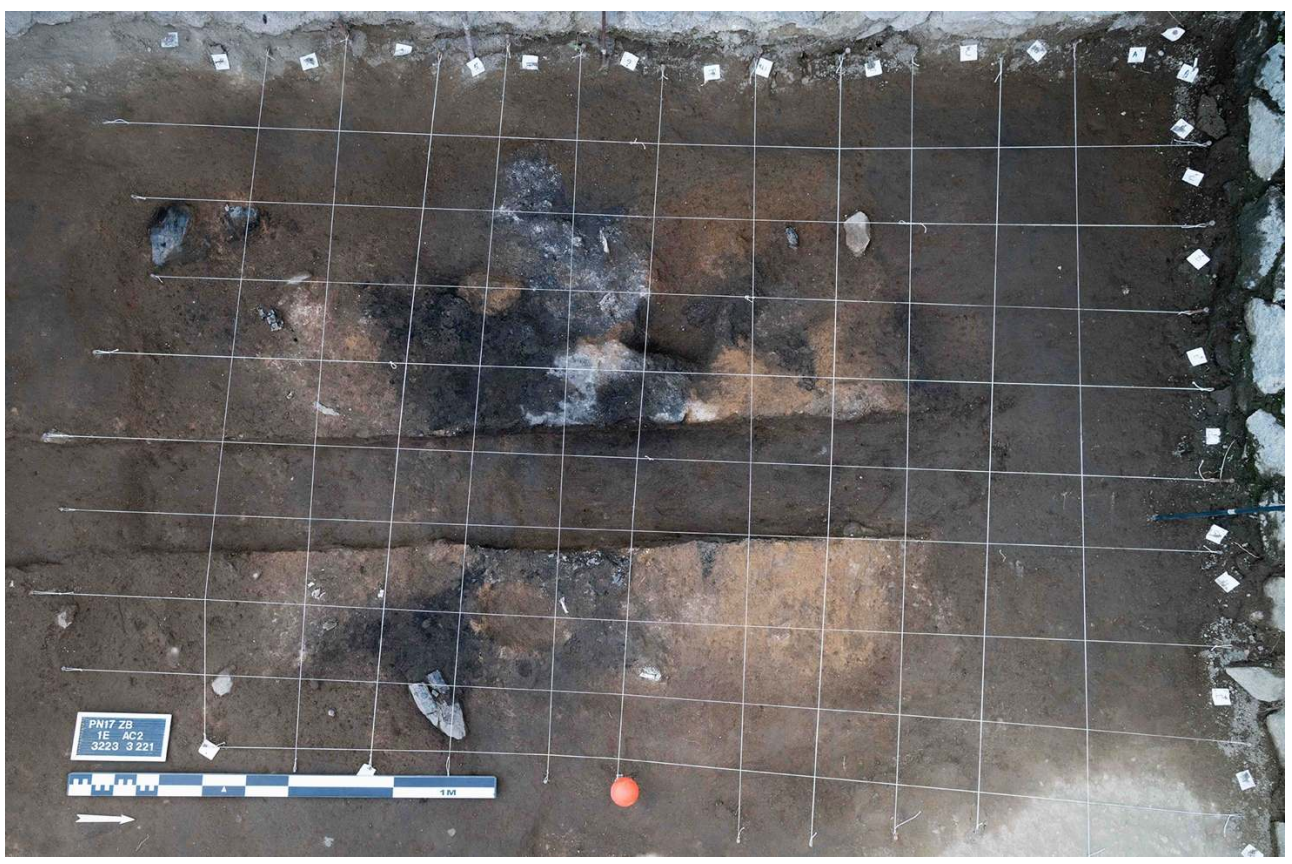

Cl. F. Giraud/@ MAPN. Toute reproduction, par quelque moyen que ce soit, reste interdite.

Fig. 9 - Plan général de l'enclos 1E.

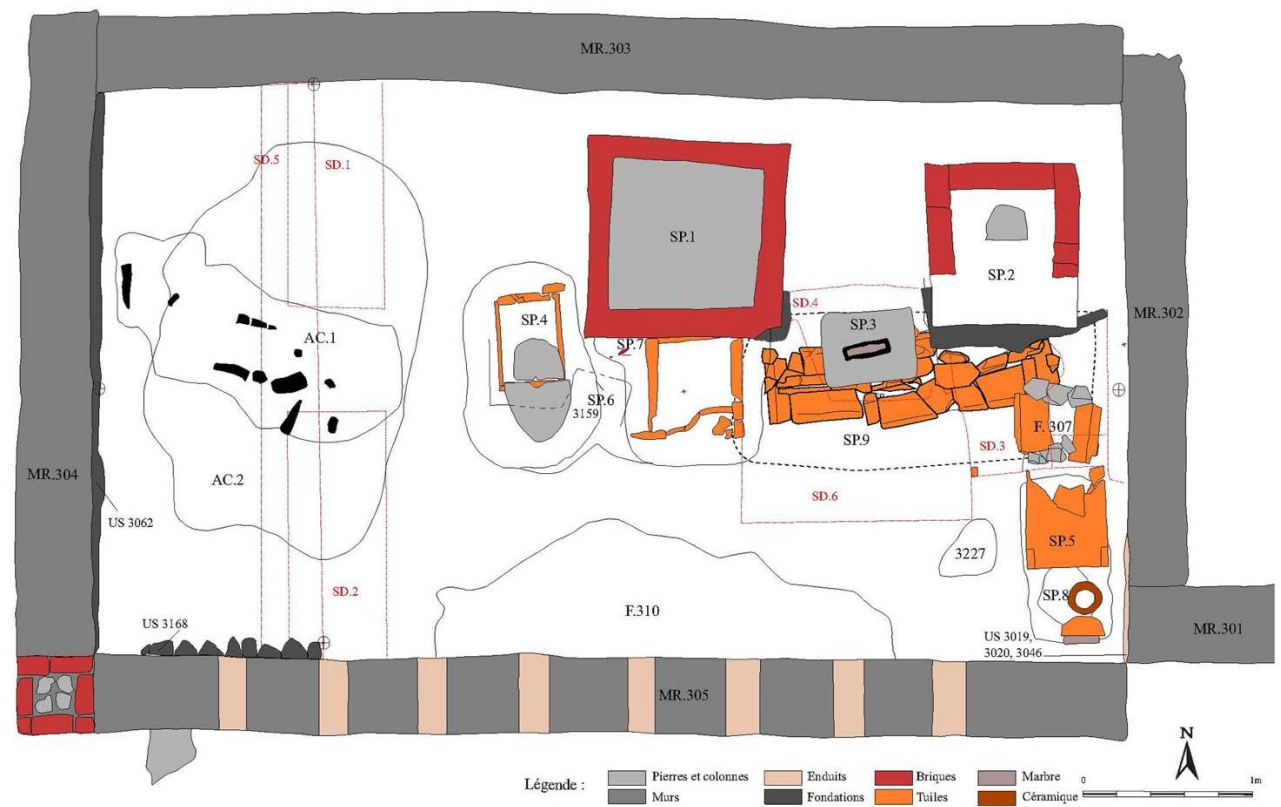

Relevé : A.-S. Vigot, S. Thorimbert, A. Boucherie, Ph. Brunner ; DAO : A.-S. Vigot ; @ MAPN. Toute reproduction, par quelque moyen que ce soit, reste interdite.

Enfin, des observations complémentaires sur la sépulture en bâtière découverte en 2016 ont été conduites. Il s'agit vraisemblablement d'une tombe-bûcher dont la fouille sera réalisée en 2018. Y était déposée une lampe à huile datée du premier siècle avant notre 
ère. Les données stratigraphiques invitent à y reconnaitre la plus ancienne tombe de l'enclos 1E, lequel en contient un minimum de 9.

L'enclos $1 F$ se situe dans la continuité du $1 \mathrm{E}$ (fig. 10). La dédicace indique que le monument a été fondé par une affranchie des Veranii : C(aio) Veranio Q(uinti) f(ilio) / Rufo IIvir(o) / Verania Q(uinti) l(iberta) Clara optimo / patrono sibi et suis. La campagne 2017 a livré de nombreux résultats. Les niveaux profonds ont pu être observés en divers endroits. Plusieurs structures mal identifiées y sont attestées. Surtout, un premier état de l'enclos - remblais et sol d'occupation - a pu être observé. Il pourrait alors correspondre au premier état de la construction sur lequel s'est superposé le monument édifié par Verania Clara. Aux deux angles de façade, une borne inscrite portant le même texte indique que le premier enclos a été donné à Quintus Veranius par décret des décurions. Si la datation de la transaction reste à préciser, les indices recueillis permettent de proposer pour l'instant l'époque augustéenne : les autorités de la cité ont alors entrepris la restructuration de la route de Nocera et l'installation de lots funéraires sur les abords, ceux-ci ayant été soit donnés (comme le $1 \mathrm{~F}$ ), soit vendus (comme le $1 \mathrm{D}$ et $1 \mathrm{E})$. Une génération après ou peu s'en faut, une recomposition familiale conduite par Verania Clara amène à une reprise de l'enclos primitif entièrement réorganisé au profit du couple formé par Clara et Rufus. À l'intérieur de l'enclos 1F, la fouille de la tombe du duumvir a pu être achevée (SP 1F3). Dans le conduit à libations associé à cette tombe ont été découverts des os brûlés d'un immature âgé d'environ 2 ans. La tombe, composée d'un caisson formé par des tuiles posées de chant, accueillait les restes de deux individus. Sous cette tombe, une céramique brisée placée dans une fosse remplie d'une couche cendreuse contenant quelques résidus osseux a été découverte. L'ensemble correspond à une sépulture antérieure à la tombe du duumvir, Caius Veranius Rufus (SP 1F13). Juste à côté se trouve la tombe de la dédicante de l'enclos, Verania Clara, postérieure à la précédente et marquée par une stèle en marbre portant l'épitaphe : Verania Q(uinti) l(iberta) / Clara. Aucun dépôt osseux n'y a été identifié malgré une fouille soigneuse et exhaustive, ce qui amène à déduire, en attendant l'achèvement de l'étude anthropologique, que les restes osseux de Verania Clara ont été déposés dans la tombe limitrophe de Caius Veranius Rufus, cette dernière contenant effectivement les restes de deux individus. Toujours au sein de l'alignement des tombes de façade, la sépulture SP 1F4 n'a pu être intégralement fouillée. La fouille de la sépulture mitoyenne $1 \mathrm{~F} 5$ a été en revanche achevée. Elle correspond à un individu âgé, probablement de sexe féminin au regard de la présence d'une stèle en lave dotée d'un chignon. À l'intérieur du contenant funéraire en céramique, un sac contenant les restes osseux avait été déposé. Une monnaie surmontait l'ensemble (fig. 11). Légèrement en retrait de l'alignement de façade, la tombe 1F7 est marquée par une stèle en lave. Les os brûlés, préalablement rassemblés dans un sac, y ont été déposés dans une urne en céramique. Ils appartiennent à une femme âgée. Signalons également la découverte, à l'arrière de l'alignement des sépultures $1 \mathrm{~F} 1$ à $1 \mathrm{~F} 7$, d'une amphore déposée sur le flanc. Une telle structure permet de reconnaitre une sépulture d'immature qui, si elle a été programmée et mise en terre, n'a jamais reçu de corps. À proximité immédiate, une tombe d'enfant a par contre été identifiée et fouillée (SP 1F12). Dans une fosse qui recoupe les niveaux de fréquentations tardifs, un enfant âgé d'un à deux ans a été déposé. Puis un fragment d'amphore a été disposé de manière à recouvrir le corps (fig. 12). Enfin, il a été possible de fouiller une fosse dans laquelle avaient été déposés les restes osseux brûlés d'un même individu (SP 1F14). L'établissement d'un carroyage a permis de mettre en évidence l'organisation du dépôt : une partie des os a probablement été rassemblée 
dans un sac avant le dépôt, tandis que le reste de la fosse accueille le résidu du bûcher simplement déversé dans le creusement. Au total, ce sont donc au moins 13 tombes qui sont attestées dans cet enclos dont la fouille se poursuivra en 2018.

Fig. 10 - Vue générale de l'enclos 1F. À gauche, en façade, l'alignement des sépultures 1F1 à 1F7 ; à droite, à l'arrière, une série de sépulture sans marquage (1F12 et 14) et une aire de crémation appartenant à la phase antérieure.

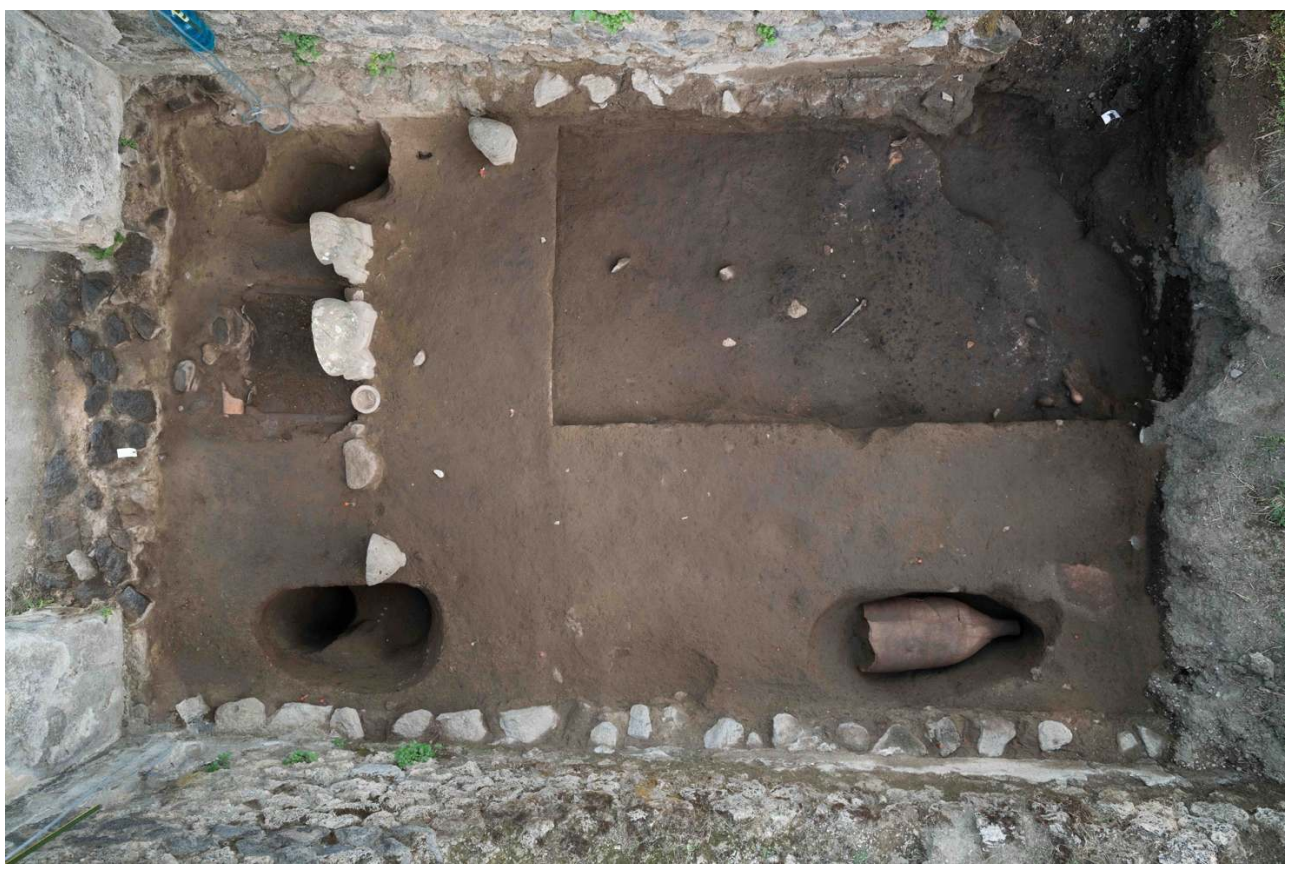

Cl. J. Laiho/@ MAPN. Toute reproduction, par quelque moyen que ce soit, reste interdite.

Fig. 11 - Le dépôt de la sépulture 1F5. Une monnaie surmonte l'amas osseux.

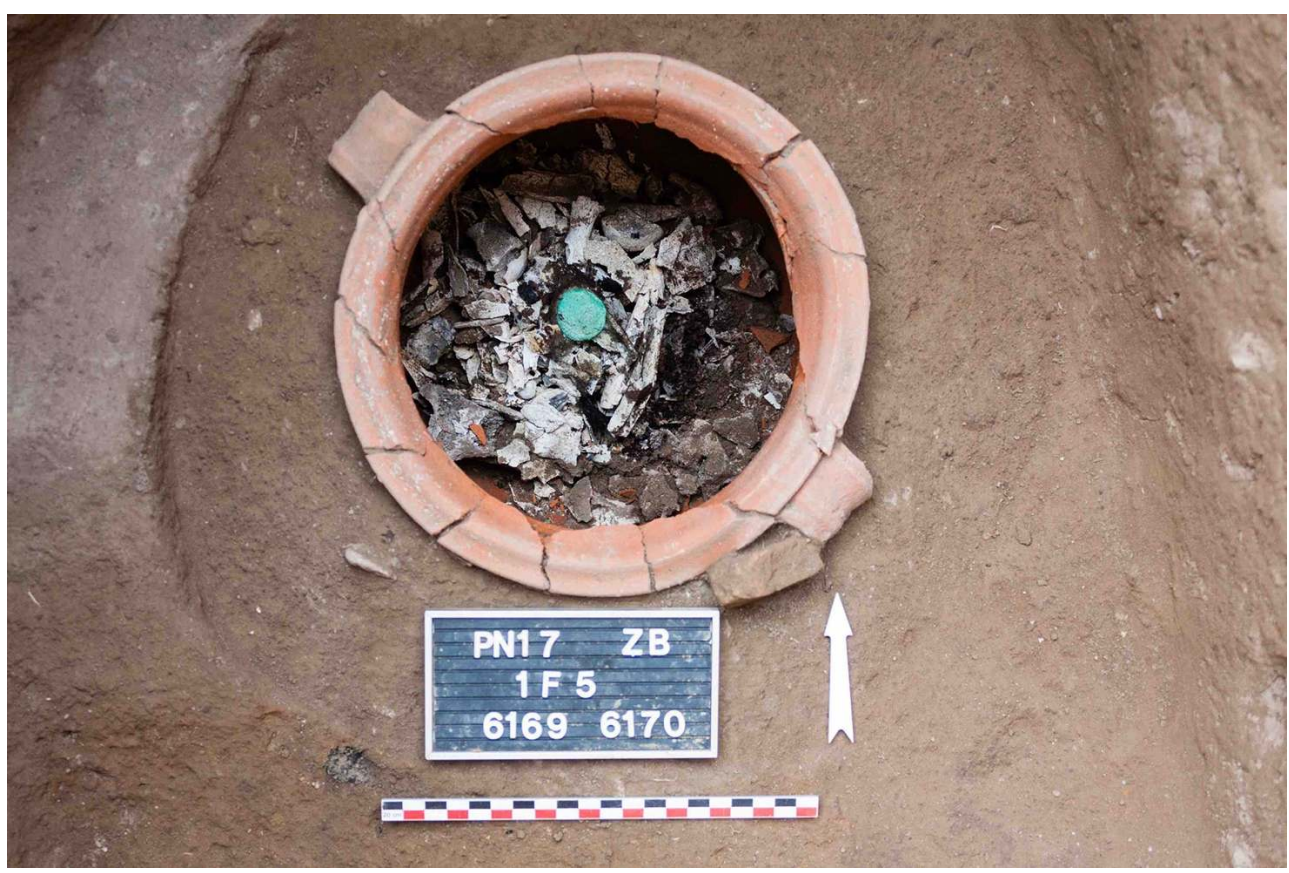

Cl. F. Giraud/@MAPN. Toute reproduction, par quelque moyen que ce soit, reste interdite. 
Fig. 12 - La sépulture 1F12.

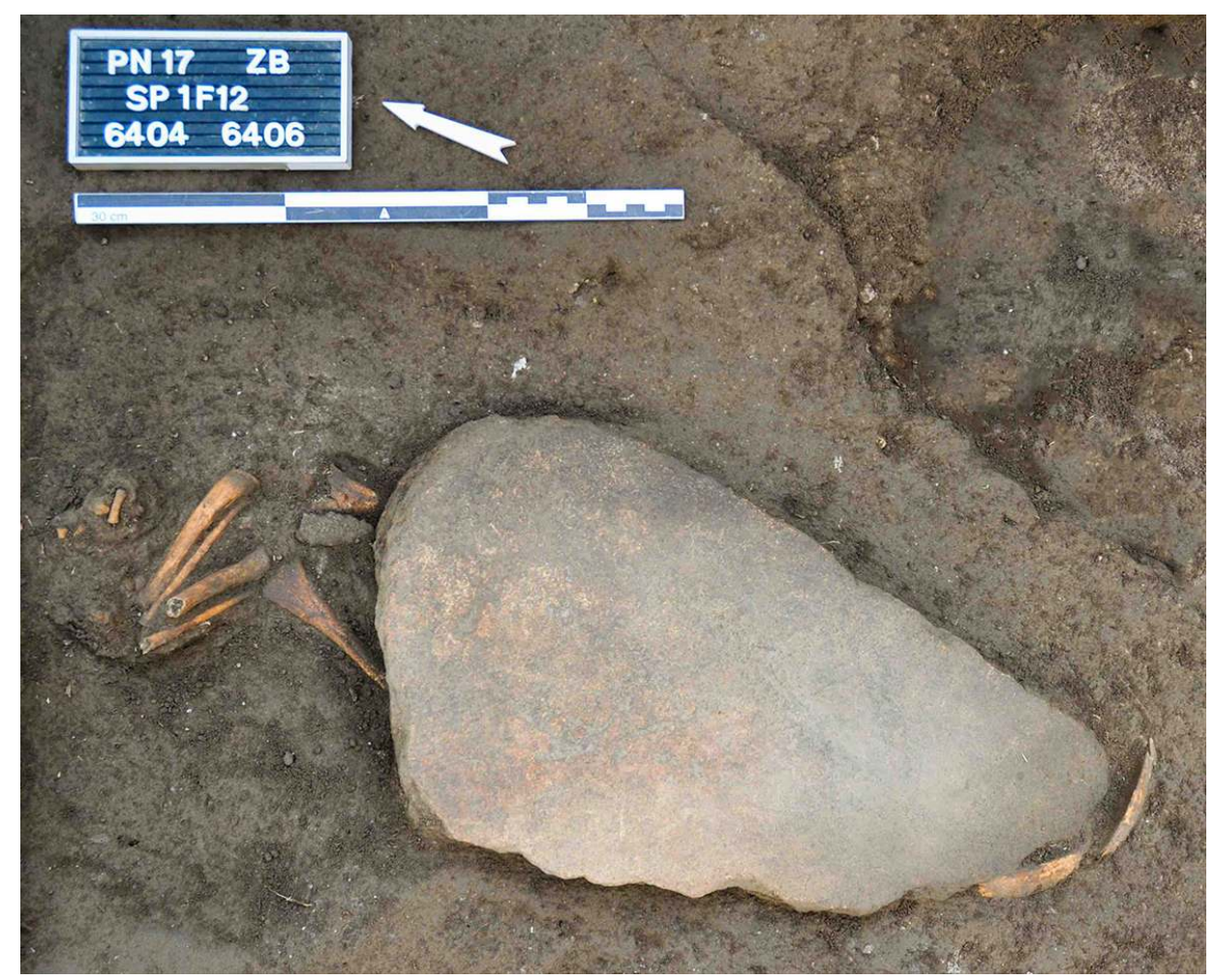

Cl. F. Giraud/@ MAPN. Toute reproduction, par quelque moyen que ce soit, reste interdite.

11 L'enclos 3E se développe au sud de la voie, au sommet d'une terrasse artificielle. Sa fouille a été amorcée en 2015. Cette année, les fouilles se sont concentrées dans la partie sud, sur les sépultures alignées contre le mur de fond de l'enclos (fig. 13). La fouille de la tombe 3E14 a été achevée. À l'origine, elle était marquée par une stèle dont le négatif de l'arrachement a pu être mis en évidence. Le conduit à libations est toujours présent. Les os brûlés étaient déposés dans un contenant funéraire en céramique. Au fond de celui-ci se trouvait une monnaie. L'an passé, une bague à chaton en or ainsi qu'un balsamaire et une petite bouteille en terre cuite avaient été retrouvés dans le comblement de la tombe. À la tombe 3E5 est associée une stèle en lave dépourvue de chignon. Les os brûlés y étaient contenus dans une urne en céramique. Une monnaie surmontait le dépôt osseux. Un premier examen invite à attribuer la sépulture à un individu adulte de sexe masculin. Cette tombe est recoupée par une tombe plus récente partiellement fouillée en 2016, SP 3E17. Dans cette dernière, les os brûlés - dont l'étude n'a pas encore été réalisée étaient déposés dans une urne en céramique au fond de laquelle se trouvait une monnaie. Le conduit à libation était posé sur le couvercle qui s'est brisé sous son poids et s'est donc enfoncé à l'intérieur de l'urne. Une dernière sépulture a été fouillée, SP 3E6. Elle est associée à une stèle en marbre à chignon. Sous une dalle percée d'une échancrure, le contenant funéraire est une urne en céramique dont le couvercle s'est brisé. Un conduit à libations situé à l'aplomb de l'échancrure est présent. Il est constitué d'un petit entonnoir en plomb prolongé par une longue tubulure qui pénètre à l'intérieur de l'urne (le couvercle s'est brisé et affaissé sous son poids (fig. 14). Dans celle-ci, les os brûlés correspondent manifestement à un individu adulte de sexe féminin. Une monnaie était 
présente au sein de l'amas. Certaines de ces sépultures recoupent une aire de crémation disposée au centre de l'enclos. Celle-ci devrait être fouillée en 2018.

Fig. 13 - Photogrammétrie de la partie sud de l'enclos 3E.

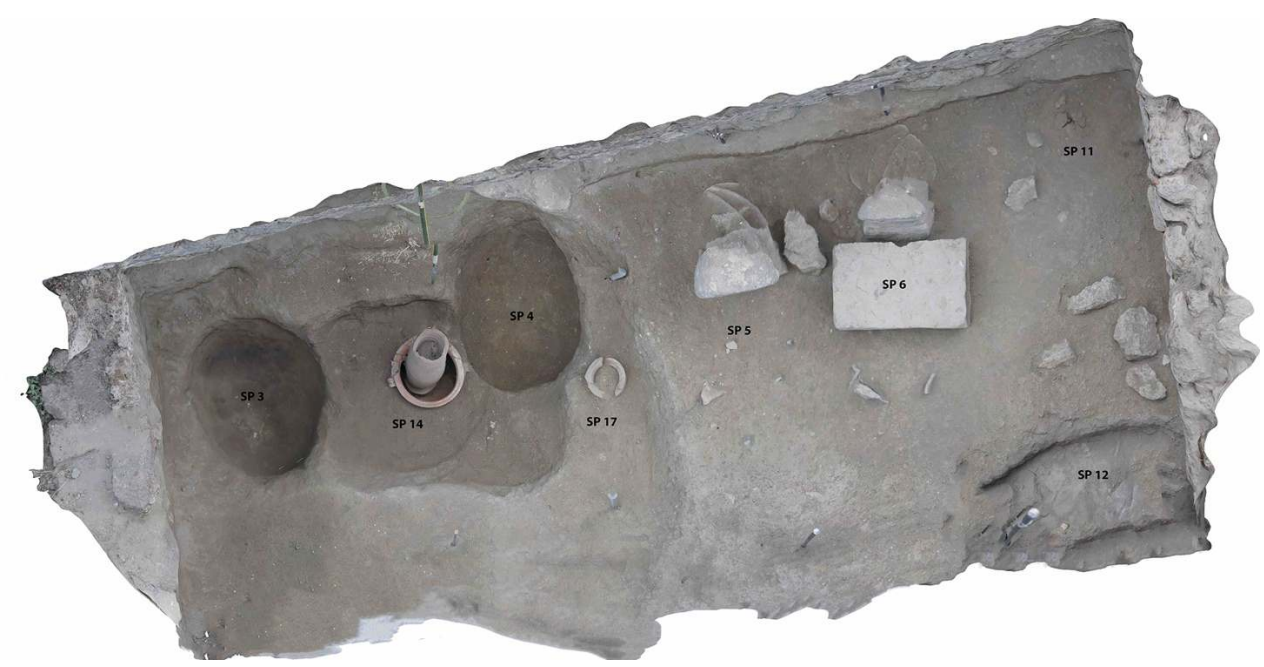

Photogrammétrie : G. Sachau-Carcel ; cl. : A. Lambert, S. Dal Col/@ MAPN. Toute reproduction, par quelque moyen que ce soit, reste interdite.

Fig. 14 - La sépulture 3E6. Le tube à libations en plomb est resté en place malgré l'effondrement du couvercle de l'urne.

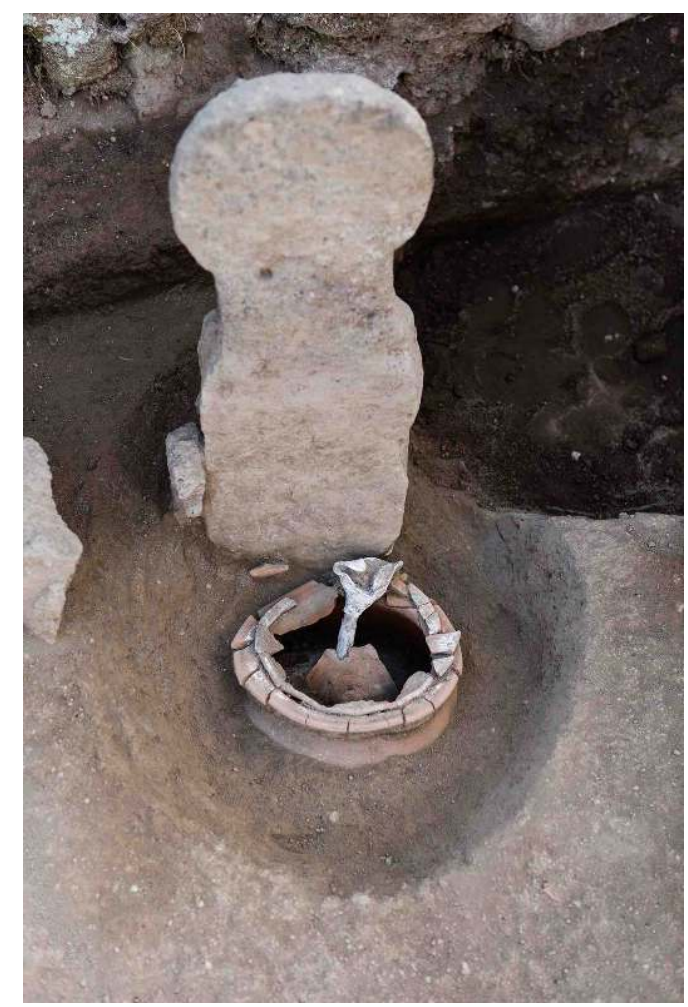

Cl. F. Giraud/@ MAPN. Toute reproduction, par quelque moyen que ce soit, reste interdite. 
12 Au total, cette campagne a livré de nombreuses informations sur la constitution du paysage funéraire et la genèse des différents enclos étudiés. La fouille d'une vingtaine de tombes, de plusieurs aires de crémation et des sols apportent de nombreuses informations sur les gestes pratiqués autour de la mort. Signalons enfin que la première semaine a été consacrée à la réalisation d'une série de prospections géophysiques, révélant des structures déjà enfouies en 79 , notamment une série de probables enclos funéraires disposés sur un terre-plein entre les monuments ES3 et ES5 ${ }^{2}$. Dans ce même temps, plusieurs études de matériel ont été réalisées : céramique, os travaillés, lampes à huile (fig. 15) et verre (fig. 16). Pendant la fouille, le matériel céramique a fait l'objet d'un premier travail d'analyse - notamment les urnes et marmites utilisées pour les amas osseux (fig. 17); les études carpologiques et archéozoologiques ont également été avancées.

Fig. 15 - Secteurs A et B : panel des lampes et décors par périodes chronologiques.

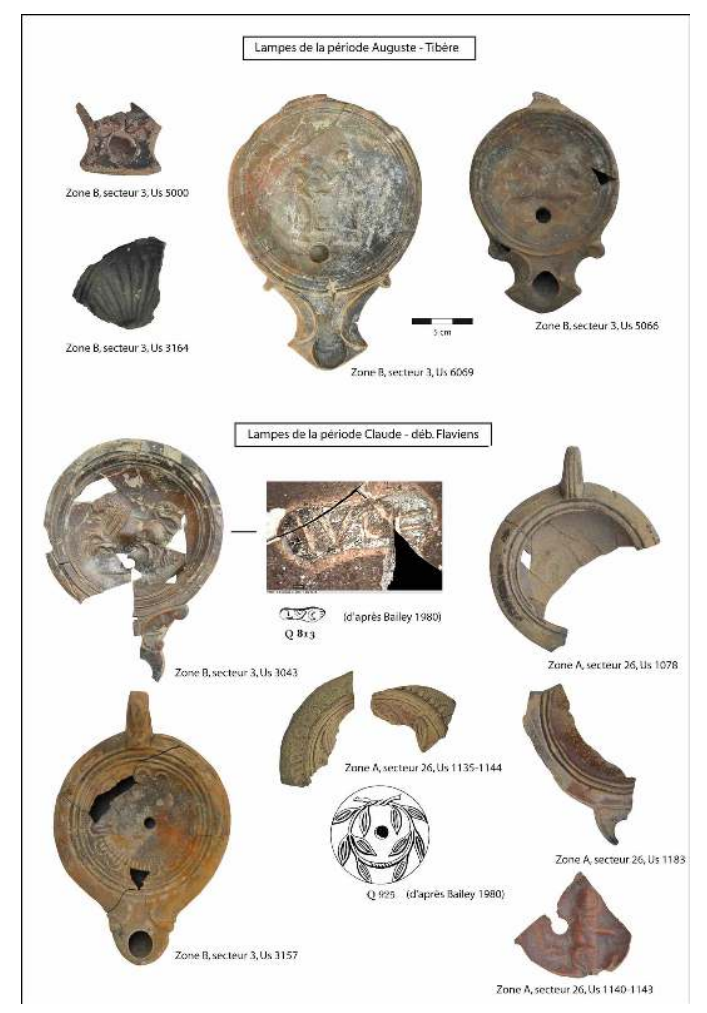

Cl. et DAO Cl. Malagoli/@ MAPN. Toute reproduction, par quelque moyen que ce soit, reste interdite. 
Fig. 16 - Planche du mobilier en verre de l'enclos 26a et de l'aire 25c.

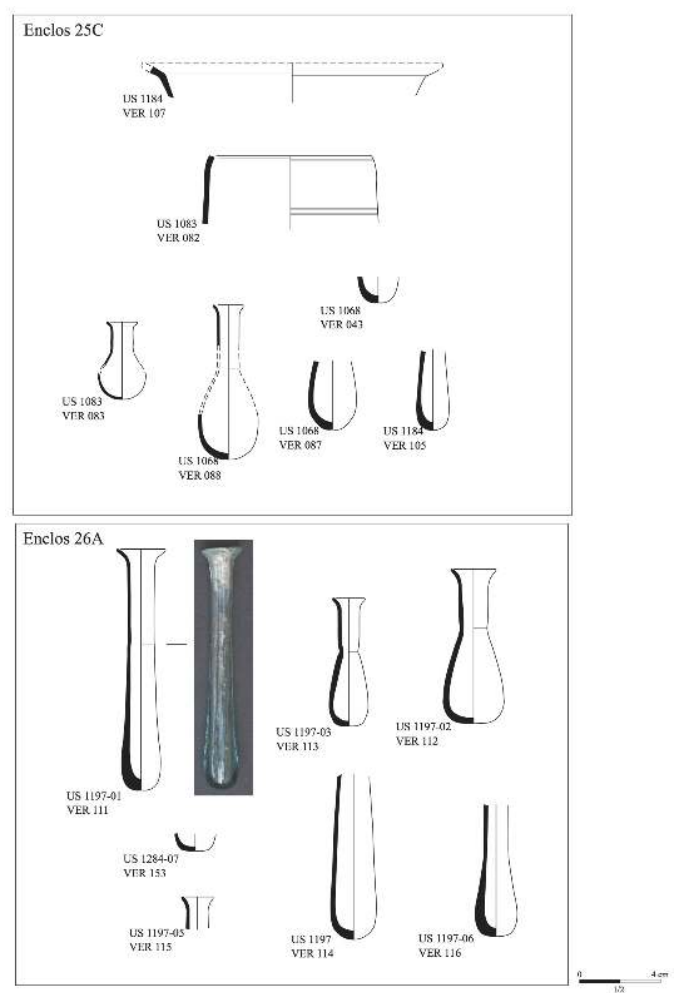

DAO : L. Robin/@ MAPN. Toute reproduction, par quelque moyen que ce soit, reste interdite.

Fig. 17 - Les urnes cinéraires étudiées en 2017.

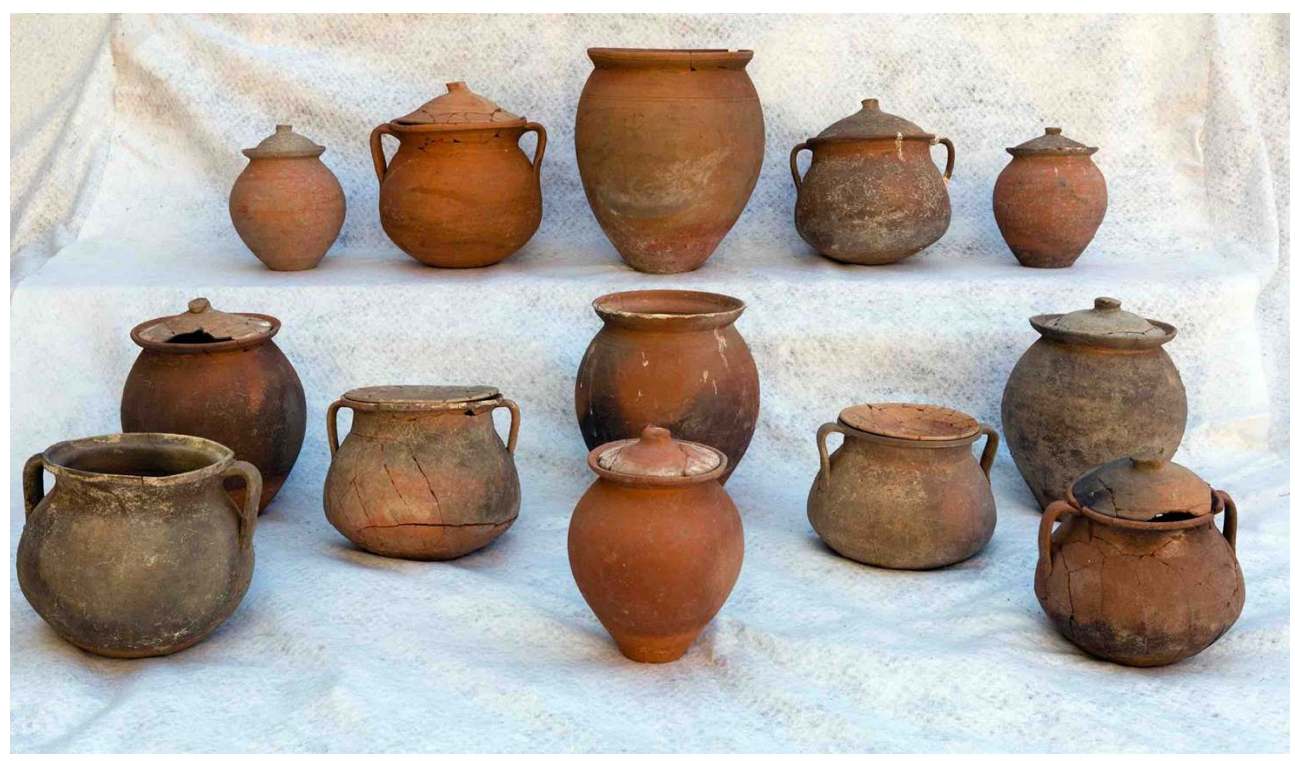

Cl. F. Giraud/@ MAPN. Toute reproduction, par quelque moyen que ce soit, reste interdite. 


\section{BIBLIOGRAPHIE}

D'Ambrosio - De Caro 1987 = A. D'Ambrosio, S. De Caro, La necropoli di Porta Nocera. campagna di scavo 1983, dans P. Zanker, H. von Hesberg (dir.), Römische Gräberstrassen: Selbstdarstellung - Status Standard. Kolloquium in München vom 28. bis 30. Oktober 1985, Münich, 1987, p. 199-228.

Duday - Van Andringa 2017 = H. Duday, W. Van Andringa, Archaeology of memory: about the forms and the time of memory in one necropolis of Pompeii, dans J. Knust, C. Moser (éd.), Ritual matters. Material residues of ancient religion, Rome, 2017, p. 73-86.

Van Andringa et al. 2013 = W. Van Andringa, H. Duday, S. Lepetz, D. Joly, T. Lind et al., Mourir à Pompéi : fouille d'un quartier funéraire de la nécropole romaine de Porta Nocera (2003-2007), Rome, 2013 ( Collection de l'École française de Rome, 468).

\section{NOTES}

1. http://www.pompeiisites.org/Sezione.jsp?titolo=Pompei\%2C+necropoli+di+Porta +Nocera\&idSezione $=7111$

2. Cette prospection a été réalisée par l'Institut archéologique autrichien, sous la direction de Stefan Groh (ÖAI).

\section{INDEX}

institutions École française de Rome, université de Lille 3 (Centre de recherches Halma UMR 8163 du CNRS), Société archéologique Éveha International, en collaboration avec l'Institut universitaire de France, l'Institut archéologique autrichien de Vienne (ÖAI) et le soutien du Parco archeologico di Pompei.

Index géographique : Pompéi

\section{AUTEURS}

\section{WILLIAM VAN ANDRINGA}

Université Lille 3 - william.va[at]free.fr

\section{THOMAS CREISSEN}

Éveha International, université de Tours - thomas.creissen[at]eveha.fr

\section{HENRI DUDAY}

CNRS, université de Bordeaux - henri.duday[at]u-bordeaux.fr 